\title{
Domain-Aware Dependency Parsing for Questions
}

\author{
Aparna Garimella* \\ Adobe Research \\ garimell@adobe.com
}

\author{
Laura Chiticariu \\ IBM Research \\ chitidus.ibm.com
}

\author{
Yunyao Li \\ IBM Research \\ yunyaoli@us.ibm.com
}

\begin{abstract}
Parsing natural language questions in specific domains is crucial to a wide range of applications from question-answering to dialog systems. Pre-trained parsers are usually trained on corpora dominated by non-questions, and thus perform poorly on domain-specific questions. Retraining parsers with domain-specific questions labeled with syntactic parse trees is expensive, as these annotations require linguistic expertise. In this paper, we propose an automatic labeled domain question generation framework by leveraging domain knowledge and seed domain questions. We evaluate our approach in two domains, and release the generated question datasets. Our experimental results demonstrate that auto-generated labeled questions indeed lead to significant $(4.9 \%-$ $9 \%$ ) increase in the accuracy of state-of-the-art (SoTA) parsers on domain questions.
\end{abstract}

\section{Introduction}

Understanding questions is the first step towards building accurate and reliable natural language interfaces. Recent works on Google Assistant, or IBM Watson focus on building domain-specific conversational agents. In this paper, we focus on syntactic parsing of domain-specific questions, which is crucial in domain-specific agents. The accuracy of syntactic parsers is known to depend on the syntactic similarities between the training data and application text. However, questions are often underrepresented in classic training corpora. In Penn TreeBank, only $0.5 \%$ of sentences from the Wall Street Journal are questions, with majority being rhetorical in nature, and those occurring in conversations (starting with interrogatives wh-/how, imperatives show me, name, or yes/no questions) are heavily underrepresented. Recognizing this search. problem, Judge et al. (2006) introduced QuestionBank, a labeled corpus of 4,000 general questions.

However, domain-specific questions are often underrepresented in general purpose question corpora, leading to their poor parsing performances; e.g., in Show me Neil's insider transactions since 2011, a SoTA parser (Nivre et al., 2016a) trained on Universal Dependencies (UD) English TreeBank (Silveira et al., 2014) and QuestionBank attaches since to show, instead of transactions, causing the system to misinterpret Neil's insider transactions since 2011, to all his transactions. In Will it rain tomorrow by noon?, tomorrow is attached to rain with a wrong dependency relation (dobj instead of nmod:tmod), causing the system to miss the temporal aspect of the question.

A natural solution to obtain accurate domainspecific parsers is to train them on domain-specific corpora. However, obtaining domain-specific questions is difficult. Moreover, annotating questions for parse trees is tedious, prone to errors and inconsistencies, and requires linguistic expertise. Petrov et al. (2010) proposed uptraining, training a parser on the output of a slower, more accurate parser. For acceptable performance, the unlabeled corpus must be large (100,000 questions). Our method is applicable when such a large corpus is not available. Inspired by (Wang et al., 2015) who showed that semantic parsers can be built "overnight" using domain expertise, we seek to reduce the effort required to handle a new domain using domain knowledge: (1) a domain schema modeling the concepts and relationships in a domain, and (2) a knowledge base of data instances that populate the schema (Hamon et al., 2017; Julien Gobeill and Ruch, 2015; Damljanovic et al., 2010). To the best of our knowledge, this is the first work to use domain knowledge to improve syntactic parsing.

This paper makes two main contributions. (1) We propose a framework to automatically generate 


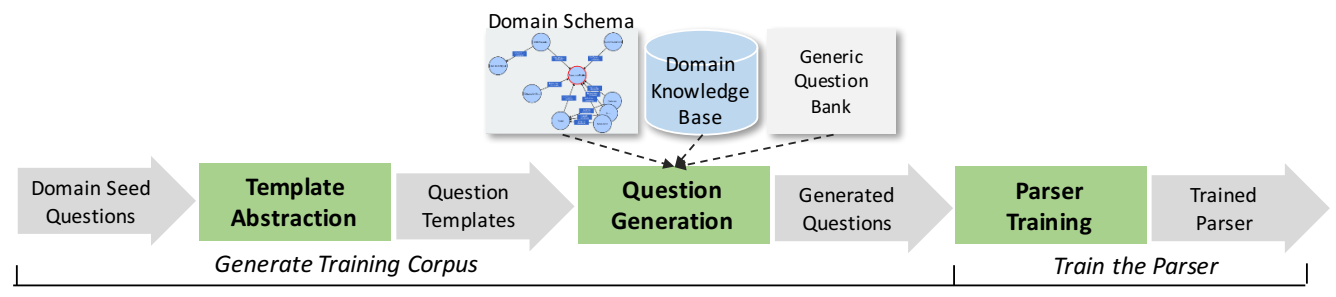

Figure 1: Overview of our approach.

a large training corpus of domain-specific questions for syntactic parsing in English, from a few seed domain questions using domain knowledge. We evaluate our approach in general (Weather) and specialized (Finance) domains. The parsers trained on the data augmented with our method have significant improvements $(4.9 \%-9 \%$ LAS, $4.8 \%-8.8 \%$ UAS) over those trained on UD Treebank and QuestionBank. Our method is robust to small seed, improving accuracy with as few as 10 seed questions. (2) We release the datasets and generated questions to the community. ${ }^{1}$

\section{Question Generation}

We automatically generate large training corpora by combining (1) domain seed questions labeled with syntactic dependencies using the Universal Dependencies v1.4 (Nivre et al., 2016b) guideline, and (2) domain knowledge. We use a two-stage pipeline: For each seed question, a question template is created that maintains its general structure, but abstracts away details of specific entities. Next, new questions are generated by automatically filling the templates with new entities obtained using domain schema and knowledge base. The domain schema is further annotated to ensure naturalness of generated questions (Figure 1).

\subsection{Template Abstraction}

Given labeled seed, this stage involves abstracting templates from their parse trees. We focus on two entity types, based on the dependency relation of nodes to their parent in parse trees of questions:

Subject Entity (qsubj) is the subject in a question. In $w h$-interrogatives, it occurs as the nominal subject (nsubj) of root. (QENT1 in Fig. 2a). In imperatives, it occurs as the direct object (dobj) of the imperative verb. (QENT 1 in Fig. 2b).

\footnotetext{
${ }^{1}$ https://github.com/System-T
}
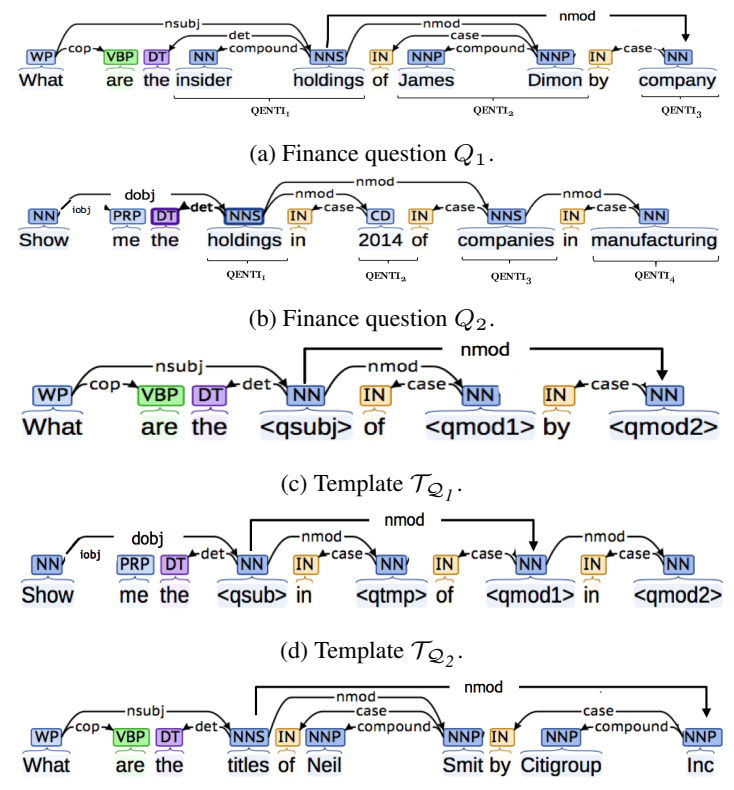

(e) Generated question $Q_{1}^{N}$.

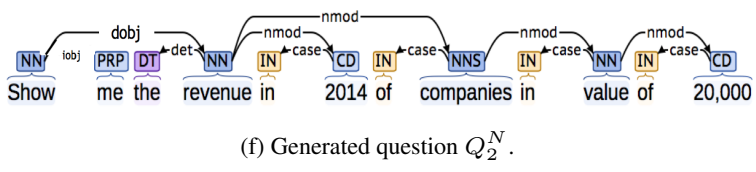

Figure 2: Finance questions $(\mathrm{a}, \mathrm{b})$, their templates $(\mathrm{c}, \mathrm{d})$ and examples of generated questions (e,f).

Modifier Entity (qmod) is a (noun phrase) node that modifies another node. In parse trees, they usually relate via an nmod dependency. ${ }^{2}$ In Fig. 2a, 'James Dimon' and 'company' are gmod entities of 'insider holdings'. Given a question, its template has qsubj and qmod entities replaced by placeholders. Algorithm 1 details template abstraction.

Domain Schema and Knowledge Base We assume domain schema as a set of classes with properties, relations between classes, and knowledge base conforming to the schema with few data instances; e.g., title is a property of class AssignmentHistory with instances 'CEO' and

\footnotetext{
${ }^{2}$ We also account for other dependencies (nmod:poss, appos, xcomp) modifying qsubj.
} 
'COO' in the Finance domain. Figure 5 in Section $B$ shows an example schema for financial domain and a few data instances in a knowledge base $\mathcal{K B}$ conforming to this schema.

\subsection{Template Filling}

Given a template, questions are generated by systematically filling qsub $j$ and qmod with new values obtained from domain schema and knowledge base. The main challenges include: (1) which new values are suitable for filling? and (2) how to automatically construct parse trees for generated questions? To address the first challenge, we propose template replacement heuristics: qsubj is replaced with properties of a class, while gmod is replaced with properties of other classes in relation to that class. Further, we introduce one-level nesting on the filled qmod by expanding them using the relative pronoun whose (further details in Section C).

We address the second challenge by constructing parse trees for template fillers as follows. (1) For each class property, we manually provide the parse tree. This incurs a small one-time effort proportional to the size of the domain schema, which is small compared to the knowledge base. (2) For each instance of a class property, we automatically generate the parse tree by making the last word as root, and attach the preceding words (Fig. 3a). (3) For qmod, we construct relative pronoun expansion node by attaching to it the parse tree of a property of a related class with tag acl:relcl and whose. Fig. 3b expands qmod $_{1}=$ Person:name='Neil Smit' using the parse tree of Holding:value with a prepositional attachment for a value (i.e., 20,000). The preposition is changed to an appropriate copular verb (is, are).

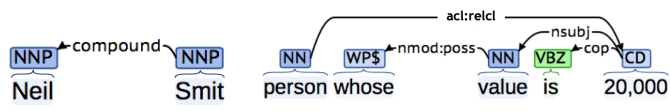

(a)

(b)

Figure 3: Parse trees for template fillers based on heuristics for data instances (a), and and relative pronoun expansion (b).

Tab. 1 shows example questions generated in the Finance domain $\left(Q_{1}^{N}\right.$ to $\left.Q_{4}^{N}\right)$.

While the generated questions exhibit variety, most are unnatural; more natural formulations are $Q_{1}^{F}$ to $Q_{4}^{F}$. The common sources of unnaturalness are the following.

Incorrect preposition for qmod In $Q_{1}^{N}$, the original preposition by is incorrect for the choice of qsubj and qmod 2 ; it should instead be in.
Incomplete usage of dependent property In $Q_{2}^{N}$, the choice of property for qsubj is incomplete; start date is not independent by itself and should be associated with a title the person holds.

Incomplete semantics of property In $Q_{3}^{N}$, the usage of property for qsubj is misleading as it refers to the value of a company, while the intent is to query the value of holdings in the company.

Incorrect question word In $Q_{4}^{N}$, what should be replaced with who, since qsubj is filled with a person, as opposed to an object.

\subsection{Schema Annotations}

A random sample of 100 generated questions contains 68 incorrect prepositions, 47 incomplete dependents, 64 incomplete semantics, and 2 incorrect question words. We address them using simple annotations to the domain schema, provided by a domain expert, in a one-time effort that is linear with the size of the schema.

Class Relations. between classes are annotated with connective words (usually prepositions), e.g., AssignmentHistory $\stackrel{\text { in }}{\rightarrow}$ Company and AssignmentHistory $\stackrel{\text { of }}{\rightarrow}$ Person in Figure 5. This annotation addresses the incorrect prepositions for gmod (e.g. $Q_{1}^{F}$ ).

Heading Properties. are those that can be queried independently without referencing others. Each property is annotated as heading or non-heading for all the classes in the schema. While heading properties and their instances can be used to fill qsubj and qmod independently, we devise rules to use non-heading ones (Appendix D). This annotation modifies the use of non-heading property start date in $Q_{2}^{N}$ by associating it with an instance 'CEO' of heading property title in $Q_{2}^{F}$.

Class-dependency of Properties. This addresses the incomplete semantics of properties. Certain properties are ambiguous, and querying them requires specifying their class names to add context; e.g., one would ask What are the values of holdings in Citigroup? as oppose to What are the values in Citigroup? Properties in the schema are annotated as class-dependent or not. This annotation leads to $Q_{3}^{F}$, a more natural version of $Q_{3}^{N}$.

Possible Question Words. To address the incorrect question words $\left(Q_{4}^{N}\right)$, we annotate all properties and their instances with corresponding possible wh- question words $\left(Q_{4}^{F}\right)$. 


\begin{tabular}{ll}
\hline Naively generated question & Final question \\
\hline$Q_{1}^{N}:$ What are the titles of Neil Smit by Citigroup Inc? & $Q_{1}^{F}:$ What are the titles of Neil Smit in Citigroup Inc? \\
$Q_{2}^{N}:$ What are the start dates of Neil Smit by Citigroup & $Q_{2}^{F}:$ What are the start dates as CEO of Neil Smit in Citi- \\
Inc? & group Inc? \\
$Q_{3}^{N}:$ What are the values of Citigroup Inc by Neil Smit? & $Q_{3}^{F}:$ What are the values of holdings in Citigroup Inc of \\
& Neil Smit? \\
$Q_{4}^{N}:$ What are the CEOs of Citigroup Inc? & $Q_{4}^{F}:$ Who are the CEOs in Citigroup Inc? \\
\hline
\end{tabular}

Table 1: Questions generated from "What are the insider holdings of James Dimon by company" with template: "What are the qsubj of qmod 1 by qmod 2 ?"

\begin{tabular}{llrr}
\hline DATASET & DomaIN\# SENT. \\
\hline UD & UD Treebank & General & 16,622 \\
GQ & QuestionBank & General & 4,000 \\
\hline DQF $_{F}$ & Domain-specific questions & Finance & 250 \\
DQW & labeled by linguist & Weather & 250 \\
\hline
\end{tabular}

Table 2: Datasets in our experiments.

\begin{tabular}{lllll}
\hline \multirow{2}{*}{ DATASET } & \multicolumn{2}{c}{ FINANCE } & \multicolumn{2}{c}{ WEATHER } \\
\cline { 2 - 5 } & LAS & UAS & LAS & UAS \\
\hline UD & 78.44 & 80.71 & 74.26 & 78.09 \\
UD+GQ & 80.62 & 82.7 & 79.48 & 82.44 \\
UD+DQ & 85.06 & 86.96 & 79.06 & 82.26 \\
UD+N & 86.34 & 88.61 & 79.47 & 82.89 \\
UD+GQ+DQ & 86.05 & 87.88 & 81.97 & 84.42 \\
UD+GQ+N & $88.31^{*}$ & $90.41^{\dagger}$ & $84.37^{*}$ & $87.21^{\dagger}$ \\
UD+GQ+DQ+N & $\mathbf{8 9 . 6 7}^{*}$ & $\mathbf{9 1 . 5 3}^{\dagger}$ & $\mathbf{8 4 . 8 2}^{*}$ & $\mathbf{8 7 . 4 9}^{\dagger}$ \\
\hline
\end{tabular}

Table 3: Performance of Malt with different training sets. LAS with * (UAS with ${ }^{\dagger}$ ) are statistically significant compared to all other settings using McNemar's test $(p<0.05)$.

\section{Experiments and Results}

We evaluate our approach with 5-fold crossvalidation in the Finance ( 9 classes, 16 relations, 63 props., 3,028 instances) and Weather ( 9 classes, 1 relation, 85 props., 66 instances) domains.

Data. Table 2 summarizes different datasets used in our experiments. For each domain, we randomly sample 250 questions from the query logs of an internal QA system, and manually annotate them with dependency parse information $\left(\mathrm{DQ}_{\mathrm{F}}\right.$ and $\mathrm{DQ}_{\mathrm{W}}$ ). We group the questions based on their template structure, resulting in 148 and 166 unique templates for Finance and Weather, respectively. Questions corresponding to $20 \%$ templates are used for training in each fold, resulting on average in 38 and 54 seed questions from 30 and 34 templates for Finance and Weather, respectively. The remaining questions (212 and 196 questions for Finance and Weather) are used as test sets to evaluate the trained parsers. In this way, the train and test sets do not have any overlap even at the template-level.

From $\mathrm{DQ}$, we generate new questions $\mathrm{N}_{\mathrm{DQ}}$ us-

\begin{tabular}{lllll}
\hline & \multicolumn{2}{c}{ FINANCE } & \multicolumn{2}{c}{ WEATHER } \\
\cline { 2 - 5 } DATASET & LAS & UAS & LAS & UAS \\
\hline UD & 72.31 & 80.19 & 69.87 & 76.27 \\
UD+GQ & 76.41 & 84.50 & 72.76 & 78.44 \\
UD+DQ & 80.47 & 86.10 & 77.30 & 82.24 \\
UD+N $D Q$ & 80.56 & 86.34 & 77.98 & 83.07 \\
UD+GQ+DQ & $81.99^{*}$ & $87.84^{\dagger}$ & $80.44^{*}$ & $84.80^{\dagger}$ \\
UD+GQ+N & $\mathbf{8 2 . 4 3}^{*}$ & $\mathbf{8 8 . 5 4}^{\dagger}$ & $\mathbf{8 1 . 0 3}^{*}$ & $\mathbf{8 5 . 3 4}^{\dagger}$ \\
\hline
\end{tabular}

Table 4: SyntaxNet results with different training sets.

ing our approach. As the number of questions generated from each seed question is very large (140,325 for Finance), and they have similar syntactic structures, we include in $\mathrm{N}_{\mathrm{DQ}}$ a maximum of 50 questions randomly selected from those generated from each seed question (this value is chosen from validation experiments).

Models. We use two parsers with default parameters: Malt (Nivre et al., 2016a), as it is fast to train, and SyntaxNet, a SoAT neural model (Andor et al., 2016). We measure parser performance using UAS (Eisner, 1996) and LAS (Nivre et al., 2004) metrics, on the test portions of $\mathrm{DQ}_{\mathrm{F}}$ and $\mathrm{DQ}_{\mathrm{W}}$.

Effectiveness. Tables 3 and 4 shows the results of Malt trained on different combinations of datasets. SyntaxNet displays similar trend, though with lower scores. Adding GQ to UD improves performance in both domains. Adding DQ leads to greater improvements for Finance, though GQ contains many more questions than DQ. This confirms the importance of domain-specific questions in the training data for specialized domains such as Finance. Adding generated questions $\mathrm{N}_{\mathrm{DQ}}$ leads to comparable, if not better, performances over UD+GQ and UD+GQ+DQ for both domains, with small sizes (1,150 questions for Finance, 1,136 for Weather, on average), illustrating the effectiveness of our algorithm in generating labeled domain questions. $\mathrm{UD}+\mathrm{GQ}+\mathrm{N}_{\mathrm{DQ}}$ and $\mathrm{UD}+\mathrm{GQ}+\mathrm{DQ}+\mathrm{N}_{\mathrm{DQ}}$ achieve the best performance: LAS gain of $4.9 \%-9 \%$ over UD+GQ, and 


\begin{tabular}{lrr}
\hline DATASET & FINANCE & WEATHER \\
\hline UD & 64.54 & 64.56 \\
UD+DQ & 65.14 & 64.75 \\
UD+DQ+N & 66.00 & 65.26 \\
\hline
\end{tabular}

Table 5: LAS of Malt parser on GQ as test.

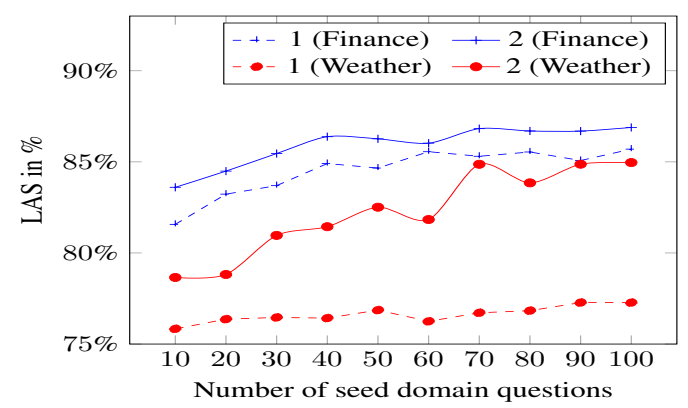

Figure 4: Malt performance trained with increasing sizes of DQ (1: $\mathrm{UD}+\mathrm{DQ}, 2$ : $\left.\mathrm{UD}+\mathrm{DQ}+\mathrm{N}_{D Q}\right)$.

$2.3 \%-3.6 \%$ over UD+GQ+DQ. This shows that our framework generates effective labeled domainspecific questions which help improve parser performances when used for training them.

Table 5 shows LAS of Malt parser trained on domain-specific seed and generated questions, and evaluated on GQ. The improvements with the addition of DQ and $\mathrm{N}_{\mathrm{DQ}}$ on general questions illustrate that our framework does not overfit to a specific domain; instead, the augmented training sets only facilitate an increase in the performances.

Effect of Schema Annotations. Schema annotation is a one-time effort and is proportional to the schema size; it required an average of 2 hours for the authors to annotate each schema. The domain understanding required for this can be acquired in a fairly small amount of time as the annotations are straight-forward, as opposed to the heavy linguistic expertise required to annotate questions with parse trees, which generally requires weeks or even months of effort to obtain a decently large training data. Moreover, large-scale human dependency tree annotations are error-prone, inconsistent and intensive, as annotators may tend to forget the many linguistic rules involved, and need to constantly ensure that the same rules are applied everywhere. Another sample of 100 questions, generated using the schema annotations, do not exhibit the anomalies listed in Section 2.2. Moreover, the inclusion of schema annotations leads to $0.4 \%-0.94 \%$ gains in LAS, thus compensating the effort required to annotate the ontology.

Robustness to Size of Domain Seed. To study the performance variation with the size of training data, we randomly sample $1 / 3^{\text {rd }}$ templates as test (49 Finance and 55 Weather questions), and train the parser on questions from remaining templates with varying sizes. Figure 4 shows LAS averaged over 5 runs with Malt in two settings: UD+DQ and $\mathrm{UD}+\mathrm{DQ}+\mathrm{N}_{\mathrm{DQ}}$. Our framework leads to significantly better performances in both domains, and even with only 10 seed questions, LAS improves by $2.03 \%$ (Finance) and $2.82 \%$ (Weather). We also note that in the Finance, adding 10 domain questions to UD leads to comparable performance to adding entire QuestionBank, and 30 questions are needed for achieve the same in Weather (UD+GQ in Table 3). As Finance is a specialized domain, $\mathrm{N}_{\mathrm{DQ}}$ from even a small seed set have a higher effect compared to a more general domain like Weather.

\section{Conclusions}

We proposed a method to automatically generate labeled domain-specific questions from small seed set using domain knowledge, to compensate for the lack of training data. We introduced ontology annotations that enhance the naturalness of the automatically generated questions. Our approach resulted in a significant increase in the LAS of $2.3 \%-2.4 \%$ over training with standard corpora and domain seed in two domains, and is robust to the seed set size. With sufficient labeled data, some of these heuristics could potentially be learned from the data. We believe our work paves way to develop domain-independent question parsing methods with very little or possibly no training data.

\section{References}

Daniel Andor, Chris Alberti, David Weiss, Aliaksei Severyn, Alessandro Presta, Kuzman Ganchev, Slav Petrov, and Michael Collins. 2016. Globally normalized transition-based neural networks. In Proceedings of the 54th Annual Meeting of the Association for Computational Linguistics (Volume 1: Long Papers), ACL 2016, pages 2442-2452, Berlin, Germany.

Danica Damljanovic, Milan Agatonovic, and Hamish Cunningham. 2010. Natural language interfaces to ontologies: Combining syntactic analysis and ontology-based lookup through the user interaction. In Proceedings of the 7th International Conference on The Semantic Web: Research and Applications - Volume Part I, ESWC'10, pages 106-120, Heraklion, Crete, Greece.

Jason M Eisner. 1996. Three new probabilistic models for dependency parsing: An exploration. In Proceedings of the 16th conference on Computational 
linguistics-Volume 1, COLING 1996, pages 340345, Copenhagen, Denmark.

Thierry Hamon, Natalia Grabar, and Fleur Mougin. 2017. Querying biomedical linked data with natural language questions. Semantic Web, 8(4):581-599.

John Judge, Aoife Cahill, and Josef Van Genabith 2006. QuestionBank: Creating a corpus of parseannotated questions. In Proceedings of the 21st International Conference on Computational Linguistics and the 44th annual meeting of the Association for Computational Linguistics, ACL 2006, pages 497-504, Sydney.

Emilie Pasche Dina Vishnyakova Pascale Gaudet Amos Bairoch Julien Gobeill, Arnaud Gaudinat and Patrick Ruch. 2015. Deep question answering for protein annotation. Database: The Journal of Biological Databases and Curation, bav081.

Joakim Nivre, Johan Hall, and Jens Nilsson. 2004 Memory-based dependency parsing. In Proceedings of the Eighth Conference on Computational Natural Language Learning (CoNLL-2004) at HLT-NAACL 2004, New York City, USA.

Joakim Nivre, Johan Hall, and Jens and Nilsson. 2016a. Malt Parser, v1.9, released June 4 2016. http: // www.maltparser.org/download.html.

Joakim Nivre, Marie-Catherine de Marneffe, Filip Ginter, Yoav Goldberg, Jan Hajic, Christopher D. Manning, Ryan McDonald, Slav Petrov, Sampo Pyysalo, Natalia Silveira, Reut Tsarfaty, and Daniel Zeman. 2016b. Universal dependencies v1: A multilingual treebank collection. In Proceedings of the Tenth International Conference on Language Resources and Evaluation, LREC 2016, pages 1659-1666, Portorož, Slovenia.

Slav Petrov, Pi-Chuan Chang, Michael Ringgaard, and Hiyan Alshawi. 2010. Uptraining for accurate deterministic question parsing. In Proceedings of the 2010 Conference on Empirical Methods in Natural Language Processing, EMNLP 2010, pages 705713, Cambridge, MA, USA.

Natalia Silveira, Timothy Dozat, Marie-Catherine de Marneffe, Samuel Bowman, Miriam Connor, John Bauer, and Christopher D. Manning. 2014. A gold standard dependency corpus for English. In Proceedings of the Ninth International Conference on Language Resources and Evaluation, LREC 2014, pages 2897-2904, Reykjavik, Iceland.

Yushi Wang, Jonathan Berant, and Percy Liang. 2015. Building a semantic parser overnight. In Proceedings of the 53rd Annual Meeting of the Association for Computational Linguistics and the 7th International Joint Conference on Natural Language Processing (Volume 1: Long Papers), ACL IJCNLP 2015, pages 1332-1342, Beijing, China.

\section{A Template Abstraction}

We refer to qmod entities that have temporal values as qtmp.

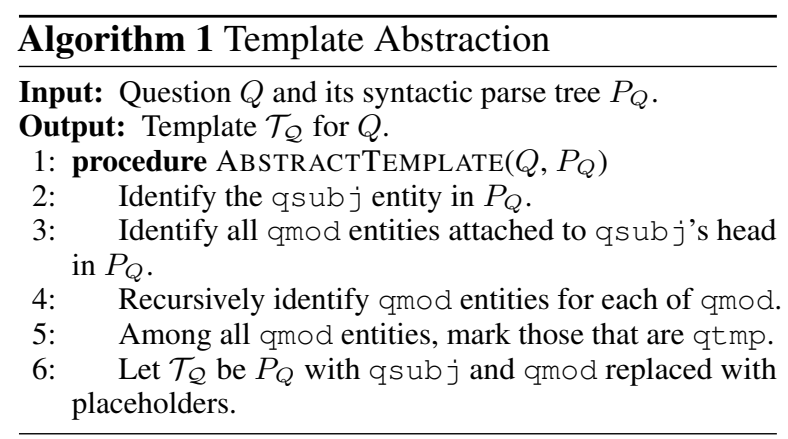

\section{B Domain Schema}

Fig. 5 shows an example schema for financial domain and a few data instances in a knowledge base $\mathcal{K B}$ conforming to this schema. The schema models a subset of the Finance domain, including people and companies with some of their related classes $\mathcal{R}$ such as address and financial metrics. It also models several relations of interest including a person's holding in a company, and a person's job assignment as an officer of a company. Ellipses represent classes; rectangles show class properties and their data types; For example, title is a property of class AssignmentHistory with instances 'CEO' and 'COO' in Finance domain.

We denote properties as class:property and their instances as class:property= 'instance'. For classes with a property name, we consider instances of name as instances of the class (e.g., 'Citigroup Inc' is an instance of class Company). Labeled arrows denote relations between classes. For example, in Fig. 5, AssignmentHistory is related to Company and Person, modeling the relationships between a person's assignment within a company; e.g., Neil Smit is the CEO of Citigroup.

\section{Template Filling}

We detail the template replacement heuristics for each entity type here. If sufficient labeled data is available, some of these heuristics could potentially be learned from the data.

Entity qsubj can be filled with either (1) a class property, ${ }^{3}$ or (2) an instance of a class property of type string, provided that the property is not name and the instance is not a

\footnotetext{
${ }^{3}$ If the part-of-speech tags of the property and qsubj do not match, appropriate changes are made to the template.
} 


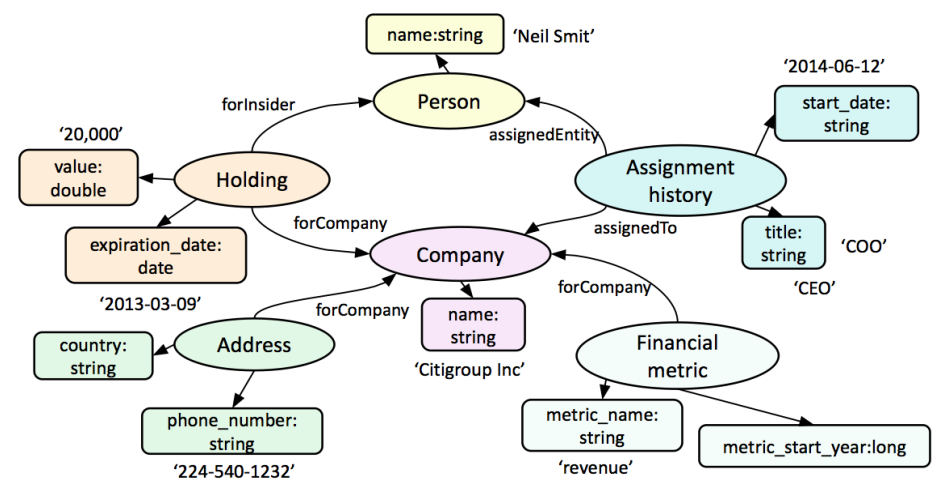

Figure 5: Domain schema for the finance domain, and example data instances. Classes, properties (their data types), and relations between classes are shown as ovals, rectangles and labeled arrows. Data instances are shown next to corresponding properties in quotes.

proper noun. For example, qsubj in $\mathcal{T}_{\mathcal{Q}_{1}}$ can be filled with AssignmentHistory:title or AssignmentHistory: ' $\mathrm{CEO}$ ' resulting in What is the title of ...? and Who is the CEO of ...? The proper noun restriction avoids generating meaningless questions such as What is the 224-540-1232 of ..? The restriction on name and string type avoids questions such as What is the Citigroup Inc ...?, and What is the 20,000 of ..?

Entity qmod is filled based on the QENT it modifies. If QENT is a class property, qmod is filled with an instance of a related class. In $\mathcal{T}_{\mathcal{Q}_{1}}$, when qsub $j$ is filled with AssignmentHistory: title, the gmod 1 slot can be filled with Person: name= 'Neil Smit', resulting in What are the titles of Neil Smit ..? If QENT is a class instance, it is changed to the name of the class, and gmod is filled with a $V_{p}$ of property $p$ of a related class. For example, in $\mathcal{T}_{\mathcal{Q}_{2}}$, if gmod 1 is filled with company: name= 'Citigroup Inc', it will be changed to 'company', and gmod 2 can be filled with $V_{p}$ value of 20,000 of Holding:value as Holding is related to Company.

Relative pronouns for qmod expansion. To generate more complex questions, we introduce onelevel nesting on an already filled gmod by expanding it using relative pronoun whose. If gmod is not a $V_{p}$ of any property $p$, we replace it with its corresponding class name, and attach a relative modifier clause using $V_{p}$ of a property $p$ of one of its related classes. In $\mathcal{T}_{\mathcal{Q}_{1}}$, when qmod ${ }_{1}$ is filled with Person: name $=$ 'Neil Smit', the relative pronoun expansion of qmod ${ }_{1}$ is person whose value is 20,000 using related class Holding of Person.

Entity qtmp is always retained as temporal values do not change the syntactic context of questions. In Fig. 2f, see $Q_{2}^{N}$ generated from $\mathcal{T}_{\mathcal{Q}_{2}}$.

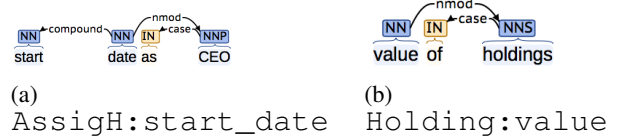

Figure 6: Templates for non-heading property (a) and classdependency (b).

\section{Heading Property Heuristics}

While heading properties and their instances can be used to fill QENT entities independently, we adopt the following rules for non-heading properties:

- For classes with a single heading property with an instance (e.g. AssignmentHistory:title with 'CEO'), the instance is used along with a non-heading property to query it using prepositional connectives which are also annotated along with heading properties (AssignmentHistory:start_date as CEO). The QENT replacement is automatically constructed by attaching the heading property's instance as nmod using prepositional connective to the parse tree of non-heading property (Fig. 6a). - For classes with more than one heading property, only they can be queried (Holding:value, Holding:expiration_date). E.g., what is the value of ..?, what is the expiration date of ...? 